\title{
Effect of metal hydride properties in hydrogen absorption through 2D- axisymmetric modeling and experimental testing in storage canisters
}

\author{
Raquel Busqué ${ }^{a^{*}}$, Ricardo Torres ${ }^{b}$, Joan Grau ${ }^{b}$, Vicente Roda ${ }^{a}$, Attila Husar ${ }^{a}$ \\ a Institut de Robòtica i Informàtica Industrial, CSIC-UPC Llorens i Artigas 4-6, 08028 Barcelona, Spain \\ ${ }^{b}$ Fluid Mechanics Department. EEBE UPC - Barcelona Tech. C/ Eduard Maristany, 10-14, 08019 Barcelona, Spain
}

\begin{abstract}
A two-dimensional axisymmetric model is developed to study the hydrogen absorption reaction and resultant mass and heat transport phenomena inside a metal hydride canister. The model is compared against published literature and experimental data. Experimental tests are performed on an in-house fabricated setup using different cooling scenarios. An extensive study on the effects of the metal properties on charging performance is carried out through nondestructive testing (NDT). Results show that the properties that most influence the charging performance are: absorption rate constant $\left(C_{a}\right)$, activation energy $\left(E_{a}\right)$ and thermal conductivity $\left(k^{m}\right)$. A Higher porosity $(\varepsilon)$ reduces charging time and amount of hydrogen stored while a higher cooling level produces a faster charging process. These results can be used to select metal hydride materials but also to estimate the metal hydride internal state and the process can be used for future evaluation of metal hydride degradation.
\end{abstract}

Keywords: Hydrogen storage; Metal hydride; Hydrogen absorption; Two-dimensional axisymmetric simulation; Experimental testing

\section{Nomenclature}

$\begin{array}{llll}C_{a} & \text { Absorption rate constant }\left[\mathrm{s}^{-1}\right] & \text { Subscripts } & \\ E_{a} & \text { Activation Energy }\left[\mathrm{kJ} \mathrm{mol}^{-1}\right] & 0 & \text { Initial } \\ \vec{g} & \text { Gravity }\left[\mathrm{m} \mathrm{s}^{-2}\right] & \mathrm{emp} & \text { Empty } \\ h & \text { Convection coefficient }\left[\mathrm{W} \mathrm{m}^{-2} \mathrm{~K}^{-1}\right] & e q & \text { Equilibrium } \\ H / M & \text { Hydrogen to metal ratio } & \mathrm{m} & \text { Mass (source term) } \\ k & \text { Thermal conductivity }\left[\mathrm{W} \mathrm{m} \mathrm{m}^{-1}\right] & \text { sat } & \text { Saturation } \\ K & \text { Permeability }\left[\mathrm{m}^{2}\right] & T & \text { Energy (source term) } \\ m & \text { Mass }[\mathrm{kg}] & \text { in } & \text { Inlet } \\ M & \text { Molecular weight }\left[\mathrm{kg} \mathrm{mol}^{-1}\right] & \mathrm{atm} & \text { Atmospheric } \\ n & \text { Number of moles }[\mathrm{mol}] & \mathrm{amb} & \text { Ambient }\end{array}$

\footnotetext{
*Corresponding autor: Raquel Busqué (rbusque@iri.upc.edu)

Tel: +34 934015751

Fax: +34 934015750

Co-authors: Ricardo Torres (ricardo.torres@upc.edu)

Joan Grau (joan.grau@upc.edu)

Vicente Roda (vroda@iri.upc.edu)

Attila Husar (ahusar@iri.upc.edu)
} 


$\begin{array}{llll}\vec{n} & \text { Normal vector } & & \\ p & \text { Pressure }[\mathrm{Pa}] & \text { Superscripts } & \\ R & \text { Universal gas constant }\left[\mathrm{J} \mathrm{mol}^{-1} \mathrm{~K}^{-1}\right] & \text { eff } & \text { Effective } \\ S & \text { Source term } & g & \text { Gas } \\ t & \text { Time }[\mathrm{s}] & \mathrm{m} & \text { Metal } \\ T & \text { Temperature }[\mathrm{K}] & \text { ref } & \text { Reference } \\ \vec{u} & \text { Velocity field }\left[\mathrm{m} \mathrm{s}^{-1}\right] & & \\ & & \\ G r e e k s & & \\ \Delta H & \text { Reaction enthalpy }\left[\mathrm{kJ} \mathrm{mol}^{-1}\right] & \\ \Delta S & \text { Entropy }\left[\mathrm{mol}^{-1} \mathrm{~K}^{-1}\right] & \\ \varepsilon & \text { Porosity } & \\ \theta & \text { Absorbed hydrogen fraction } & \\ \mu & \text { Dynamic viscosity }\left[\mathrm{kg} \mathrm{m}^{-1} \mathrm{~s}^{-1}\right] & & \\ \rho & \text { Density }\left[\mathrm{kg} \mathrm{m}^{-3}\right] & & \end{array}$

\section{Introduction}

As energy consumption increases, society, industry and governments have become aware of the necessities to invest in sustainable energies that can decrease the problems associated with the use of fossil fuels and other non-renewable sources. Recent studies [1] show that the use of hydrogen as an energy vector can aid to satisfy the present and future energy demands without additional carbon emissions.

Due to its calorific value and being environmentally friendly, hydrogen energy has become a possible replacement for fossil fuels, depending on how it is produced. As hydrogen is abundant in water and in various hydrocarbons, it could be easily produced using renewable energy sources. Hence, it can facilitate the transition from the present fossil fuel energy economy to a future hydrogen based economy $[2,3]$.

A major concern that needs to be addressed to make hydrogen technology economically feasible is the safe storage of hydrogen in vessels with the desirable weight, volume and cost. The future of hydrogen as an energy vector will strongly depend on these technologies [4].

Hydrogen has the particularity of having a very low energy density per unit of volume, so it requires a lot of volume to store a large amount of energy in gaseous state. This is a problem when there is a limited amount of storage space available, for example in automotive applications.

Hydrogen can be stored in many ways, for example as a compressed gas inside high-pressure tanks. In order to satisfy space limitations and energy requirements depending on the application, pressures are typically around $70 \mathrm{MPa}$. These levels of pressure cause safety concerns and a high energy cost when pressurizing the tanks.

Liquid storage in cryogenic tanks constitutes another possible alternative. The problem is that it would require extremely low temperatures and also a cooling system that would have high energy costs as well. 
Hydrogen can also be stored as an absorbed element in solid porous materials (metal hydride) [5]. Metal hydride based hydrogen storage offers certain advantages compared to high-pressure gaseous or cryogenic liquid storage systems in terms of compactness, storage at ambient conditions, possibility of tailoring metal hydrides to suit different temperature-pressure requirements, as well as being inherently safe because hydrogen is stored at low pressure [68]. The disadvantage is that it offers low energetic density per unit of mass due to the weight of the metal itself. But on the contrary, it offers a high energy density per unit of volume.

A primary application of hydrogen storage is proton exchange membrane fuel cells (PEMFCs), which use pure hydrogen as their fuel [9].

Prior studies show that reactions in metal beds can be effectively modeled as a two-dimensional problem. Jemni and Ben Nasrallah developed two-dimensional heat and mass transfer models for hydrogen absorption and desorption respectively $[10,11]$. Their results show that the difference between the solid and hydrogen temperatures is negligible, except for some limited areas close to the gas outlet and tank wall, so the local thermal equilibrium hypothesis can be used. In a subsequent study, Jemni et al. [12] conducted an experimental test to determine the effective thermal conductivity, the equilibrium pressure, and reaction kinetics to validate the theoretical model. A good agreement between measured and theoretical results was obtained.

Muthukumar et al. $[13,14]$ numerically investigated the performance of a metal hydride storage device at different supply pressures, cooling fluid temperatures, overall heat transfer coefficients and bed thicknesses using a two-dimensional heat and mass transfer model. An analysis of the performance of a storage system with embedded cooling tubes during the absorption of hydrogen was also carried out. Different container geometries, depending upon the number and arrangement of cooling tubes inside the hydride bed were simulated.

Also Jiao et al. [15] studied the effects of cooling level and charging pressure in a twodimensional axisymmetric model. They concluded that increasing the heat transfer coefficient (cooling the bottle) and charging pressure improves the hydrogen absorption.

Nam et al. [16] developed a three-dimensional model to study the hydrogen absorption reaction, heat and mass transport phenomena in a metal hydride hydrogen storage tank. $\mathrm{A}$ parametric study was carried out for various designs and hydrogen feed pressures. The simulation results demonstrate that the external surface to volume ratio is one of the key factors to determine the hydrogen absorption performance, and that the use of a higher hydrogen supply pressure leads to not only rapid hydrogen charging performance but also a reduction in the cooling burden of the tank.

Chung et al. [17] investigated the influences of buffer area and heat convection. The hydrogen storage canister comprises of a cylindrical metal bed and a void buffer area above the metal. The buffer area is considered as a domain of pure hydrogen gas. Simulation results show the buffer area reduces the reaction rates by increasing thermal resistance to the heat transfer from the outside cooling/heating bath.

Regarding experimental results, Dhaou et al. [18] empirically studied hydriding/dehydriding rates and the pressure-composition isotherms for different metal hydrides under quasi- 
isothermal and variable pressure conditions. They derived empirical rate equations to describe the sorption reaction kinetics. In a later study, Dhaou et al. [19] performed some experimental tests on a metal hydride cylindrical prototype fitted with two heat exchangers. These studies were performed varying the supply pressure and the cooling temperature of the hydride bed. They concluded that at any given cooling temperature, hydrogen storage rate increases with supply pressure and also that cooling temperature has a significant effect on hydrogen storage capacity at lower supply pressures.

Although considerable efforts have been made regarding the modeling of hydrogen absorption in metal hydride vessels, a detailed analysis of its complex transport mechanisms, and mass and heat transfer is useful for efficient design, and selection of materials for any industrial application. Mathematical modeling is a tool to identify and predict the state of the metal hydride during absorption as well as to determine the relationship between different factors. The evaluation of the state of charge of a metal hydride canister is very difficult, as it strongly depends on the working conditions, quality of the metal hydride, internal state, morphology, type of metal hydride and degradation of the material and thus, an extensive study on the metal properties and working conditions, as the one presented in this paper, is required.

This work is the first stage of research initially destined to develop procedures and protocols to design a metal hydride state of charge estimator and to evaluate the degradation of the canisters in terms of total hydrogen storage capacity. Therefore, it provides an extensive study on the effects of each individual metal property through non-destructive testing (NDT). It is also important to mention that a detailed experimental setup has been designed and assembled emulating a real application using commercially available metal hydride canisters, which has not been widely investigated in prior studies.

To that end, a 2D axisymmetric numerical model has been developed and different situations have been analyzed. Starting with a basic cylindrical geometry some parametric studies have been carried out to obtain the effect of the abovementioned properties on the charging performance. Then, these results have been used to calibrate the simulation to the real practical application. Next, the outcomes have been compared with experimental data obtained in an inhouse fabricated setup. The model and the experimental protocols can be used to estimate the metal hydride internal state as well as evaluating aspects such as, the reduction of the absorption capacity due to contamination.

\section{Numerical model}

A two-dimensional axisymmetric metal hydride hydrogen storage container is modeled to analyze the key physical phenomena occurring during the hydrogen absorption process.

The hydrogen-metal reaction is the following:

$$
M+\frac{x}{2} H_{2} \leftrightarrow M H_{x}+\Delta H
$$

Where $M$ represents the metal, $M H_{x}$ is the respective hydride and $x$ the ratio of hydrogen to metal. Finally, $\Delta H$ is the heat of reaction. The hydride formation is exothermic and consequently the reverse reaction is endothermic and therefore heat is required for hydrogen release. 
Two different geometries are tested: a cylindrical shape (Fig. 1, a) to compare the numerical simulations with published literature, and a bottle-like geometry (Fig. 1, b) to match the real metal hydride vessels available in the lab. Both geometries are implemented as two-dimensional axisymmetric domains, which have two differentiated sections: the top section is a void region acting as a buffer for the hydrogen molecules and the larger bottom section is a porous region which contains the metal hydride and where the absorption takes place.
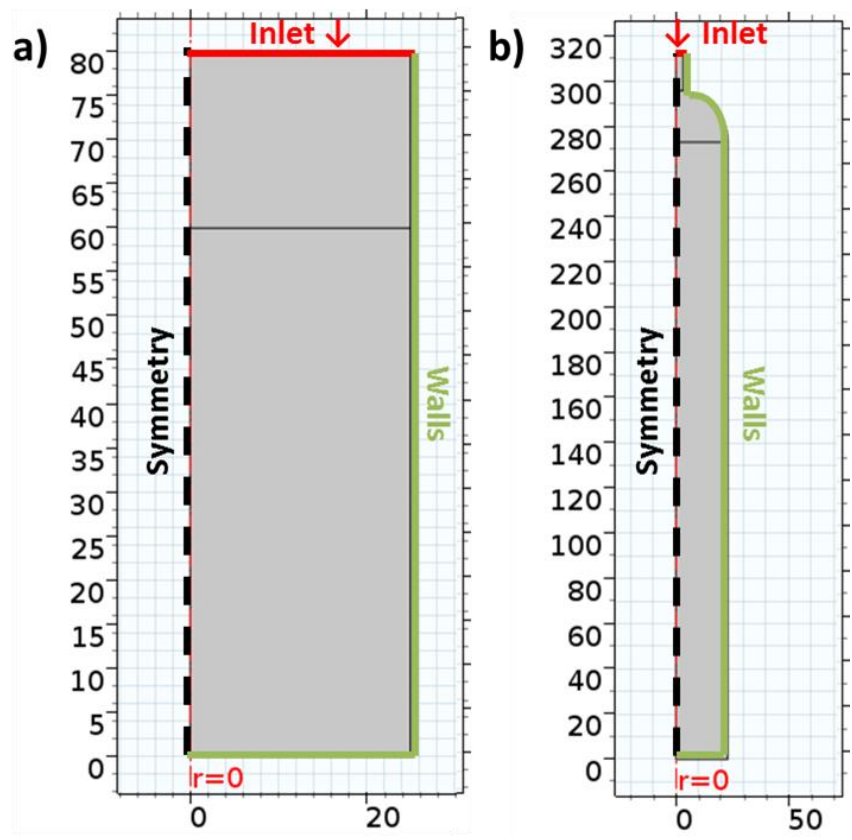

Fig. 1 Geometries with boundary conditions $(\mathrm{mm})$ : a) Literature cylindrical shape geometry,

b) Laboratory bottle-like geometry

To deal with the lack of information regarding the metal hydride inside the canisters, a detailed and systematic numerical analysis of various metal properties is performed, as well as an exhaustive study on external cooling level both numerical and experimentally.

This model is implemented using the commercially available software COMSOL Multiphysics ${ }^{\circledR}$, by means of the appropriate fluid dynamics and heat transfer modules.

\subsection{Model assumptions}

The assumptions made in the model are the following:

1. Gas phase (hydrogen) will be considered an ideal gas.

2. Powdery metal hydride will be treated as an isotropic and homogeneous porous medium.

3. Local thermal equilibrium is assumed between the solid metal and the hydrogen gas. That means that the gas and metal temperatures inside the vessel will be the same.

4. Volumetric expansion of the metal hydride during absorption will be neglected.

5. Metal hydride properties such as porosity, permeability and thermal conductivity will remain constant during the absorption process. 


\subsection{Conservation equations and source terms}

Under the assumptions above, the metal hydride container is governed by the conservation of mass, momentum and thermal energy $[17,20-23]$.

\subsubsection{Mass conservation}

For hydrogen:

$$
\varepsilon \frac{\partial \rho^{g}}{\partial t}+\nabla \cdot\left(\rho^{g} \vec{u}\right)=-S_{m}
$$

Where the gas density can be described by the ideal gas law.

For the metal hydride:

$$
(1-\varepsilon) \frac{\partial \rho^{m}}{\partial t}=S_{m}
$$

\subsubsection{Momentum conservation}

The momentum conservation will be modeled using a continuum approach through the Brinkman equations. This way the model gives an average flow rate for the total volume which is enough if the flow over large areas is considered $[24,25]$.

$$
\frac{\rho^{g}}{\varepsilon} \frac{\partial \vec{u}}{\partial t}=-\nabla p+\nabla \cdot \tau-\frac{\mu}{K} \vec{u}-\frac{S_{m}}{\varepsilon^{2}} \vec{u}+\rho^{g} \vec{g}
$$

\subsubsection{Energy conservation}

Regarding the temperature field, local thermal equilibrium has been assumed between the gas phase and the solid metal hydride; so, the energy equation can be expressed with a single temperature variable as follows [26]:

$$
\overline{\rho c_{p}} \frac{\partial T}{\partial t}+\nabla \cdot\left(\rho^{g} c_{p}^{g} \vec{u} T\right)=\nabla \cdot\left(k^{e f f} \nabla T\right)+S_{T}
$$

In Eq. (4), the effective heat capacity $\left(\overline{\rho c_{p}}\right)$, and the effective thermal conductivity $\left(k^{e f f}\right)$ can be expressed as porosity-weighted functions of the hydrogen and metal phases.

$$
\begin{gathered}
\overline{\rho c_{p}}=(1-\varepsilon) \rho^{m} c_{p}^{m}+\varepsilon \rho^{g} c_{p}^{g} \\
k^{e f f}=(1-\varepsilon) k^{m}+\varepsilon k^{g}
\end{gathered}
$$

\subsubsection{Source terms}

Mass sink term is the local hydrogen absorption rate per unit volume. Equation (7) contains three terms. The first one is the temperature dependence, which is based on an Arrhenius expression. The middle term corresponds to the pressure dependence that includes the 
equilibrium pressure explained below. And finally, the last term includes density and is added to set the maximum storage capacity of the tank.

$$
S_{m}=C_{a} \cdot \exp \left(-\frac{E_{a}}{R T}\right) \ln \left(\frac{p^{g}}{p_{\text {eq }}}\right)\left(\rho_{\text {sat }}^{m}-\rho^{m}\right)
$$

Equilibrium pressure, which is the pressure of the absorption in a state of dynamic equilibrium, indicates the direction of the reaction. If the hydrogen gas pressure is above the equilibrium pressure, the reaction proceeds to the right to form a metal hydride. If the gas pressure is below the equilibrium pressure, hydrogen is released and the metal returns to its gaseous state.

For the equilibrium pressure of hydrogen absorption two different expressions have been tested using $\mathrm{LaNi}_{5}$ as the reference metal. Equation (8) is the simplified Van't Hoff expression, and (9) is an experimental function based on $H / M$ ratio, which is the absorbed hydrogen to metal atomic ratio, and temperature presented by Dhaou et al. [18], which is a seventh-order polynomial function for hydrogen absorption on $\mathrm{LaNi}_{5}$. The overall results of this study are not independent on the metal, but the parametric studies for determining the other metal properties besides the expression for equilibrium pressure, can be used in studying other metal hydride materials.

$$
\begin{gathered}
p_{\text {eq }}=p_{\text {ref }} \cdot \exp \left(-\frac{\Delta H}{R T_{\text {ref }}}+\frac{\Delta S}{R}\right) \\
p_{\text {eq }}=p_{\text {eq }}^{r e f} \cdot \exp \left(-\frac{\Delta H}{R}\left(\frac{1}{T}-\frac{1}{T_{\text {ref }}}\right)\right) \\
p_{\text {eq }}^{\text {ref }}=-0.34863+10.1059 \cdot\left(\frac{H}{M}\right)-14.2442 \cdot\left(\frac{H}{M}\right)^{2}+10.3535 \cdot\left(\frac{H}{M}\right)^{3} \\
+4.20646 \cdot\left(\frac{H}{M}\right)^{4}+0.962371 \cdot\left(\frac{H}{M}\right)^{5}-0.115468 \cdot\left(\frac{H}{M}\right)^{6} \\
+0.00563776 \cdot\left(\frac{H}{M}\right)^{7} \\
\end{gathered}
$$

Fig. 2 Equilibrium pressure according to van't Hoff's equation (8) and experimental approximation (9) at $303 \mathrm{~K}$ 
Fig. 2 represents equilibrium pressure using both expressions. As it can be seen, pressure in the plateau region is quite similar, but the difference at the beginning and at the end is noticeably different. As hydrogen initially enters the bottle, the equilibrium pressure rises sharply. After the void volumes between the metal particles are filled, the metal hydride begins to form at constant pressure, so a plateau is observed. When the absorption is complete, another sharp pressure rise is seen as more hydrogen is added to the volume [27].

Comparing the results obtained using both expressions it has been determined that a better approach to the real behavior of the reaction is observed using the semi-empirical expression (9); therefore, this experimental function is used in this study.

Energy source term represents the release of heat through the exothermic hydrogen absorption reaction.

$$
S_{T}=S_{m}\left[\Delta H-T\left(c_{p}^{g}-c_{p}^{m}\right)\right]
$$

Finally the absorbed hydrogen fraction $(\theta)$ can be defined according to equation (12), where $(H / M)_{\text {sat }}$ is the saturated hydrogen to metal atomic ratio. Therefore $\theta$ ranges from 0 to 1 .

$$
\theta=\frac{(H / M)}{(H / M)_{s a t}}
$$

\subsection{Initial/boundary conditions and model implementation}

At first, the metal hydride container is assumed to be in thermodynamic equilibrium and hydrogen gas is initially at $1 \mathrm{~atm}$ and the vessel as is considered "empty". Also, the initial velocity of hydrogen gas is zero.

$$
T=T_{0} \quad p=p_{0} \quad \rho^{m}=\rho_{0}^{m} \quad \vec{u}=0
$$

As for boundary conditions (Fig. 1), boundary walls are assumed to be impermeable so, no-slip velocity and no-flux conditions are valid and a convection boundary condition is applied between the bottle walls and exterior air.

$$
-k^{e f f} \frac{\partial T}{\partial \vec{n}}=h\left(T-T_{a m b}\right)
$$

Inlet conditions are applied to the inlet region of computational domain according to specified temperature and pressure.

\section{Experimental characterization}

In order to compare the simulations with the experimental results and also to observe the evolution of the parameters in a practical application, the following experimental setup has been implemented $[28,29]$. 


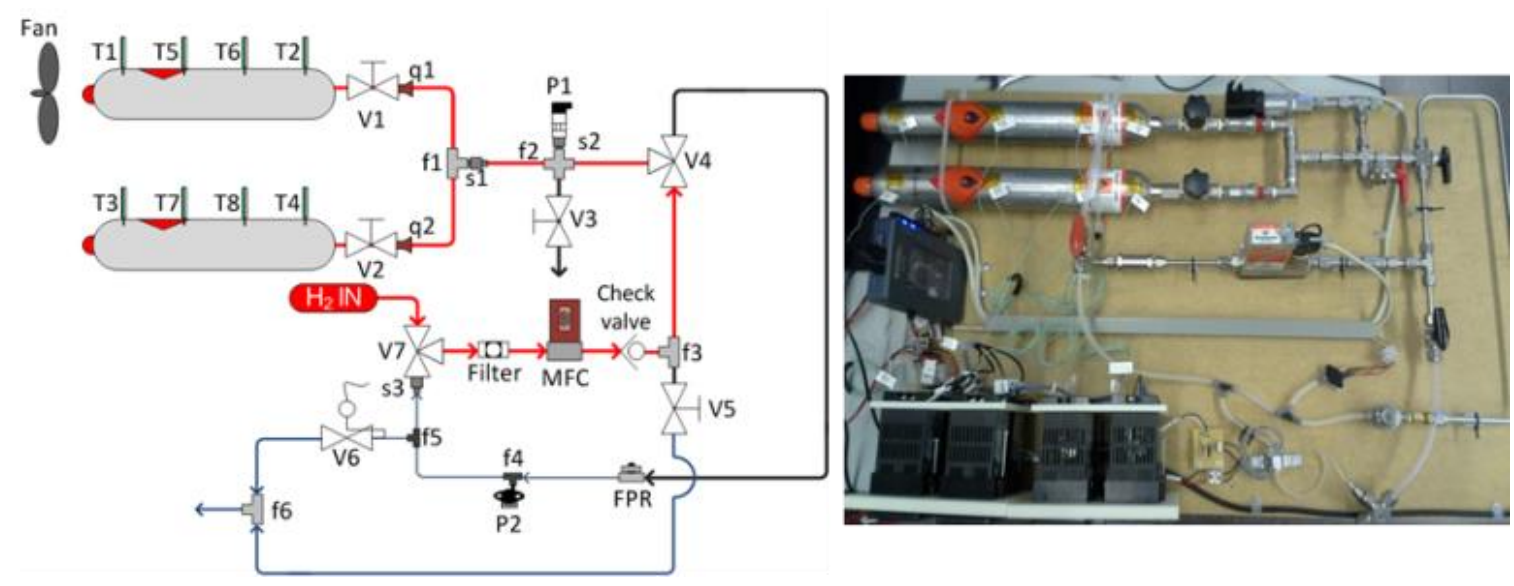

Fig. 3 Experimental setup scheme (left) and real (right)

As seen in Fig. 3, during charge (red circuit), hydrogen is supplied and goes into an EL-FLOW base Mass Flow Controller (MFC) from Bronkhorst High Tech (tests without fan using F-201C-AAD-20$\checkmark$ which has a range from 0 to $5 \mathrm{nlpm}$ and tests with fan using F-201C-AAA-22V with a range from 0 to $1.5 \mathrm{nlpm}$ ). At the beginning a MFC with an upper limit of $5 \mathrm{nlpm}$ was chosen, but in the end it was replaced for a $1.5 \mathrm{nlpm}$ MFC to obtain a more accurate result in the working region. Then it goes through a 7-micron particulate filter, to ensure that no particles enter the MFC. Next, hydrogen goes up and into the metal hydride bottles, which are Swagelok 304LHDF4-500-PD stainless steel containers filled with an unknown metal hydride. Before entering the bottles, there is a high-pressure sensor (P1). This sensor is an industrial pressure transmitter from Impress Sensors and Systems (IMP-G4002-7A4-BCV-00-000) working with pressures up to $4 \mathrm{MPa}$, and will be reading values of charge pressure. At the surface of the bottles there are also some welded tip PFA thermocouples from tc direct ( $\mathrm{T} 1$ to $\mathrm{T} 8$ ) attached in order to acquire temperature data. The control and data acquisition system is programmed in LabVIEW, and the communication between the analogical and digital signals is achieved using the National Instruments (NI) myRIO-1900 device.

Through this experimental setup, temperatures at the surface of the bottles, pressure at the inlet and entering mass flow can be measured and then used to calibrate the parameters of the computational simulation.

The experimental setup also has a fan, so that the metal hydride bottles have two different cooling scenarios: natural convection (no fan) and forced convection (fan).

In order to perform the experiments, the following protocols have been executed. First, it is necessary to make sure that the circuit is completely empty and there is no air in the tubes, so a purge with hydrogen of the whole system is required. Also, it is mandatory that the bottle that is going to be studied in each of the experiments is empty. A canister is considered empty when during discharge a steady asymptotic situation is achieved, which means that a relatively small amount of hydrogen is released (outlet mass flow below $0.15 \mathrm{nlpm}$ ). The last step before starting the charging process is to make sure that all valves are in the right position.

Once the setup protocol is completed, the charging process can start. First, the valve corresponding to the selected bottle is opened, being it V1 or V2. Then, V4 is opened in the 
direction that allows hydrogen flow from the red circuit. The last step is to release the hydrogen into the system and towards the MFC by opening V7 valve.

At this instant, the bottle is in charging mode, and the system will continue working until the hydrogen charging curve is saturated, which means that it has reached a steady asymptotic situation in which a large amount of time is required to absorb any new hydrogen molecules. At this point, the bottle is considered charged.

When the bottle is charged, the absorption process is completed and so the inlet valve V7 is closed, followed by V4 and the bottle valve (V1 or V2).

\section{Results and discussion}

\subsection{Numerical simulation cases}

This paper is divided into two parts. Firstly, the numerical model is implemented using the available data shown in Table 1 and a simple cylindrical geometry (Fig. 1, a), to compare the results with other authors' published work $[15,16]$

Table 1 Parameters used in the simulation

\begin{tabular}{lcl}
\hline Parameter & Designation & Value \\
\hline Dynamic viscosity of hydrogen & $\mu$ & $8.411 \cdot 10^{-6}[\mathrm{~Pa} \mathrm{~s}]$ \\
\hline Porosity & $\varepsilon$ & 0.63 \\
\hline Permeability of the metal hydride & $K$ & $1 \cdot 10^{-8}\left[\mathrm{~m}^{-2}\right]$ \\
\hline Thermal conductivity of hydrogen & $k^{g}$ & $0.1672\left[\mathrm{~W} \mathrm{~m}^{-1} \mathrm{~K}^{-1}\right]$ \\
\hline Thermal conductivity of the metal & $k^{m}$ & $3.18\left[\mathrm{~W} \mathrm{~m}^{-1} \mathrm{~K}^{-1}\right]$ \\
\hline Empty metal hydride density & $\rho_{e m p}^{m}$ & $4200\left[\mathrm{~kg} \mathrm{~m}^{-3}\right]$ \\
\hline Saturated metal hydride density & $\rho_{s a t}^{m}$ & $4264\left[\mathrm{~kg} \mathrm{~m}^{-3}\right]$ \\
\hline Hydrogen heat capacity & $c_{p}^{g}$ & $14.89\left[\mathrm{~kJ} \mathrm{~kg}^{-1} \mathrm{~K}^{-1}\right]$ \\
\hline Metal heat capacity & $c_{p}^{m}$ & $0.419\left[\mathrm{~kJ} \mathrm{~kg}^{-1} \mathrm{~K}^{-1}\right]$ \\
\hline Universal gas constant & $R$ & $8.134\left[\mathrm{~J} \mathrm{~mol} \mathrm{~K}^{-1}\right]$ \\
\hline Molar weight of the metal & $M^{m}$ & $432\left[\mathrm{~kg} \mathrm{kmol}^{-1}\right]$ \\
\hline Molar weight of $\mathrm{H}_{2}$ & $M^{H_{2}}$ & $2\left[\mathrm{~kg} \mathrm{kmol}{ }^{-1}\right]$ \\
\hline Atmospheric pressure (absolute) & $p_{a t m}$ & $0.1[\mathrm{MPa}]$ \\
\hline Reference pressure (absolute) & $p_{r e f}$ & $1[\mathrm{MPa}]$ \\
\hline Reference temperature & $T_{r e f}$ & $303[\mathrm{~K}]$ \\
\hline Inlet pressure (relative) & $p_{i n}$ & $1[\mathrm{MPa}]$ \\
\hline Ambient temperature & $T_{a m b}$ & $293[\mathrm{~K}]$ \\
\hline Hydrogen absorption constant on LaNi & $C_{a}$ & $59.187\left[\mathrm{~s}^{-1}\right]$ \\
\hline Hydrogen activation energy & $E_{a}$ & $21179.6\left[\mathrm{~J} \mathrm{~mol}^{-1}\right]$ \\
\hline Reaction enthalpy & $\Delta H$ & $29879\left[\mathrm{~J} \mathrm{~mol}^{-1}\right]$ \\
\hline
\end{tabular}




\begin{tabular}{lcl}
\hline Reaction entropy & $\Delta S$ & $108\left[\mathrm{~J} \mathrm{~mol}^{-1} \mathrm{~K}^{-1}\right]$ \\
\hline Saturated hydrogen to metal ratio & $(H / M)_{\text {sat }}$ & 6.5 \\
\hline Convection coefficient & $h$ & $1652\left[\mathrm{~W} \mathrm{~m}^{-2} \mathrm{~K}^{-1}\right]$ \\
\hline
\end{tabular}

Once the numerical simulation is setup and running, it is necessary to adapt it to the laboratory situation, by means of calibrating the material properties as well as the operating conditions.

\subsubsection{Sensitivity of the model to metal properties}

It is important to mention that the metal hydride bottles used in this study are commercially available, and the provider is not willing to disclose information regarding the type of metal hydride. As the container is sealed, it is not possible to determine the material properties needed to run the simulations without having to open up the canister directly. This would be a destructive test, which is not an option. One objective of this work is to determine the material properties without performing ex situ material testing, so an in-situ strategy is presented.

It is important to mention that, computational models allow studying scenarios that may be difficult or even impossible to obtain experimentally. For example, the variation of $C_{a}$ and $E_{a}$ independently from one another might not have a physical meaning, as they are both dependent on the metal hydride. However it is very interesting to visualize any specific behavior when varying input variables as an identification mechanism, as well as its effect on charging performance.

So, a parametric study is performed to identify the sensitivity of the model to the parameters that influence the metal hydride. Using this data, the model is calibrated by isolating the specific material properties that are more sensitive to the model.

After analyzing the material properties, it can be observed that there are some variables that greatly influence the performance of the metal hydride by means of shortening charge time or increasing the maximum temperature achieved, for example. But there are also some parameters that do not have a noticeable impact on the simulation results.

The parameters that have a small influence on the processes are permeability $(K)$ and activation energy $\left(E_{a}\right)$ when its value is small $\left(E_{a}<21000 \mathrm{~J} \mathrm{~mol}^{-1}\right)$.

Parameters that have a major influence on the model are the absorption rate constant $\left(C_{a}\right)$, the activation energy $\left(E_{a}\right)$ when its value is high $\left(E_{a}>21000 \mathrm{~J} \mathrm{~mol}^{-1}\right)$, the thermal conductivity of the metal $\left(k^{m}\right)$ and porosity $(\varepsilon)$. 

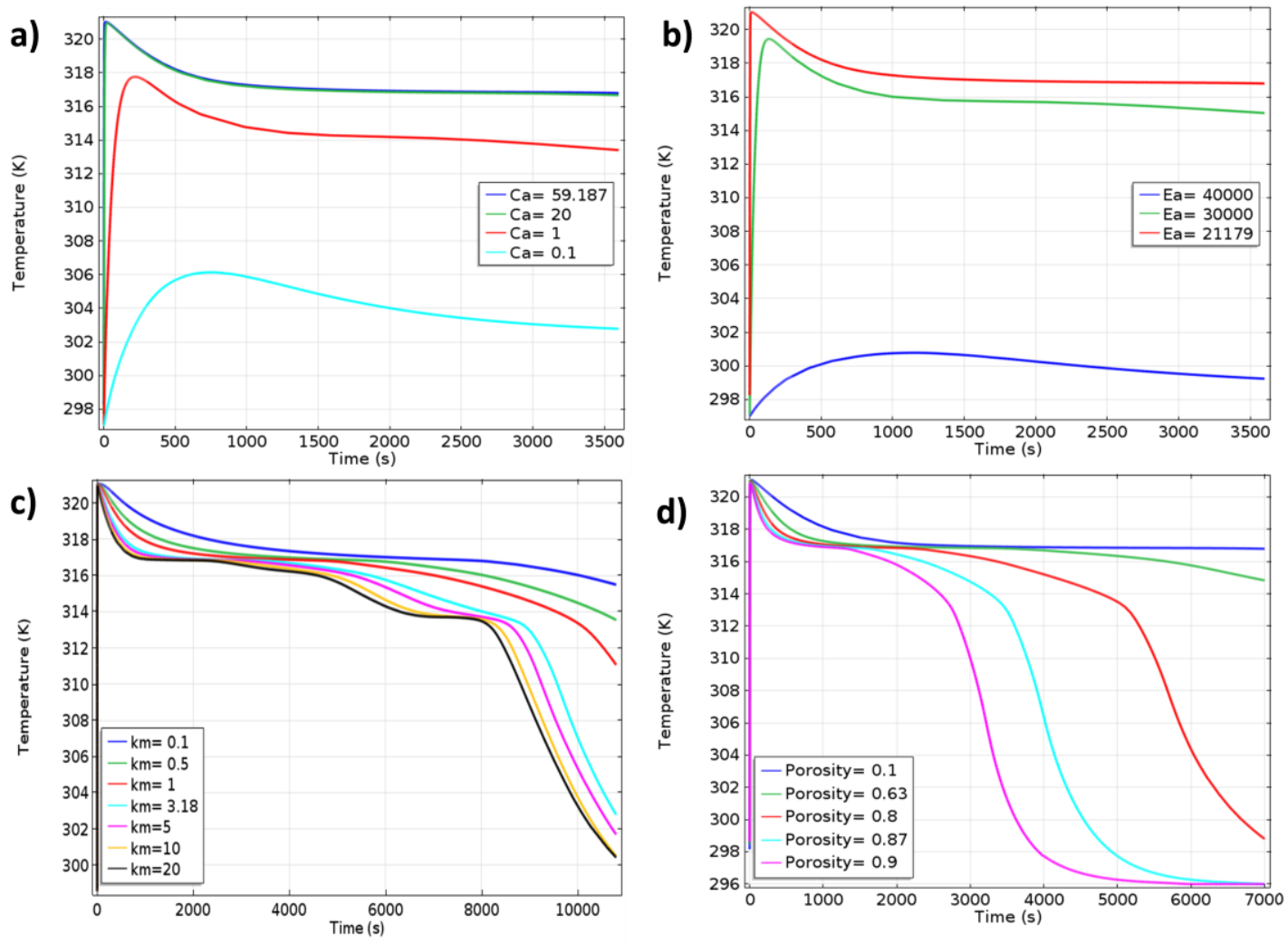

Fig. 4 Temperature evolution for different values of: a) $C_{a}\left[s^{-1}\right]$; b) $E_{a}\left[J \mathrm{~mol}^{-1}\right]$;

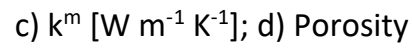

Regarding $C_{a}$ (Fig. 4, a), it can be concluded that the temperature decreases as the absorption rate constant decreases, but not in a linear rate, as there is no difference in the temperature evolution when $C_{a}=59.187 \mathrm{~s}^{-1}$ or $C_{a}=20 \mathrm{~s}^{-1}$, but there is a big difference between $C_{a}=20 \mathrm{~s}^{-1}$ and $C_{a}=1 \mathrm{~s}^{-1}$ or $C_{a}=0.1 \mathrm{~s}^{-1}$.

In Fig. 4, b, when activation energy is low, there is no noticeable difference in the temperature of the metal hydride canister. Whereas, when the activation energy is high $\left(E_{a}=40000 \mathrm{~J} \mathrm{~mol}^{-1}\right)$ a clear difference in the temperature is noticed. This change in temperature is because a large amount of energy is required to begin any hydrogen-metal absorption and as this energy level cannot be reached, absorption does not occur.

Regarding $k^{m}$ (Fig. 4, c), it can be seen that its influence on the bottle temperature is not as noticeable at the beginning as it is at the end of the charge. Initially the exothermic reaction starts increasing the temperature of the bottle, progressively the absorption process is slowed down until it finishes. When the thermal conductivity of the metal is high $\left(\mathrm{k}^{m}=20 \mathrm{~W} \mathrm{~m}^{-1} \mathrm{~K}^{-1}\right)$, it will be easier for the bottle to evacuate the excess heat produced by the reaction, hence lowering the interior bottle temperature. On the contrary, if the metal has a very poor thermal conductivity $\left(\mathrm{k}^{m}=0.1 \mathrm{~W} \mathrm{~m}^{-1} \mathrm{~K}^{-1}\right)$ it will tend to accumulate the produced heat and thus the bottle temperature will remain higher.

In Fig. 4, d, the temperature increases sharply at the beginning reaching a peak and then decreases a bit leading to a temperature plateau. This temperature level is kept more or less constant as a result of the equilibrium between heat produced by the exothermic reaction and 
heat evacuation due to convection with exterior air. As the volume of the bottle does not change, when porosity is high $(\varepsilon=0.9)$ there is less metal inside the bottle, and consequently less available metal volume for the hydrogen to fill. Therefore, the amount of hydrogen stored is lower, so the absorption process finishes sooner and the temperature decreases until it reaches ambient temperature. On the other hand, when the metal is not very porous $(\varepsilon=0.1)$, the filling process is slower, as there is a lot more metal inside the bottle and so more hydrogen is stored. The effect of the porosity on the metal hydride behavior can be useful for the design of hydrogen storage systems, with a trade-off between charge time and storage capacity.

\subsubsection{Sensitivity of the model to cooling level}

The amount of hydrogen that can be charged strongly depends on bottle temperature and inlet pressure. This study is focused on the effect of bottle temperature, through different convection coefficients, on charge rate.

A local heat transfer coefficient of $10 \mathrm{~W} \mathrm{~m}^{-2} \mathrm{~K}^{-1}$ represents the scenario in which the tank is cooled by natural convection, whilst 50 and $200 \mathrm{~W} \mathrm{~m}^{-2} \mathrm{~K}^{-1}$ represent forced air cooling scenarios and finally 600 and $1000 \mathrm{~W} \mathrm{~m}^{-2} \mathrm{~K}^{-1}$ represent liquid cooling scenarios at different levels.
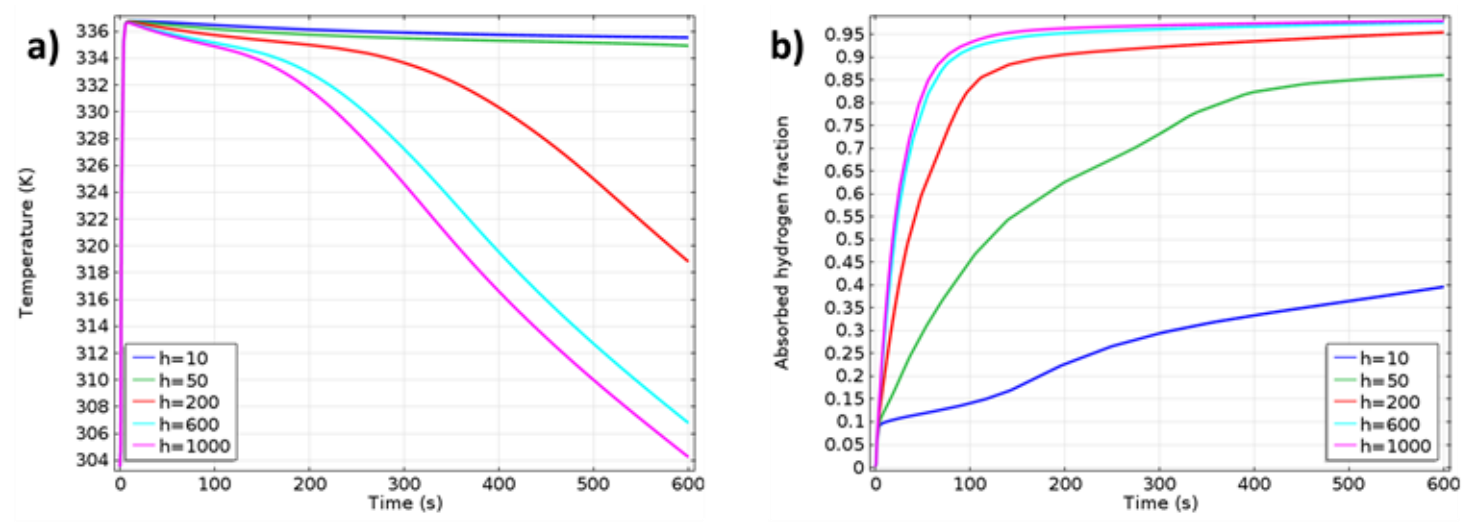

Fig. 5 Effect of heat transfer coefficient on the evolution of: a) Temperature [K];

b) Absorbed hydrogen fraction

Fig. 5, a), represents the temperature evolution at a point located at $r=15 \mathrm{~mm}$ and $z=25 \mathrm{~mm}$, under the effect of different cooling levels. Firstly, the temperature increases rapidly from $293 \mathrm{~K}$ to about $337 \mathrm{~K}$ under all cooling levels. Then, temperature is kept almost constant, with a rather small decrease for $h \leq 50 \mathrm{~W} \mathrm{~m}^{-2} \mathrm{~K}^{-1}$, because hydrogen is still being absorbed in these cases. On the other hand, temperature starts decreasing quite sharply for $h>50 \mathrm{~W} \mathrm{~m}^{-2} \mathrm{~K}^{-1}$ (forced air cooling or liquid cooling) and so the process finishes sooner when the convection coefficient is high, that is, when the bottle is cold. These results can be compared with the ones obtained by Jiao et al. [15] where it can be clearly seen that the cooling level has the same effects on the bottle temperature. The shape of both curves is very similar and the only differences are in the time scale, as some of the material properties used are different $\left(\varepsilon, k^{m}, c_{p}{ }^{g}, c_{p}{ }^{m}, T_{\text {ref }}\right)$ or unknown because the author does not provide all the information.

Regarding absorbed hydrogen fraction (Fig. 5, b), which is the ratio between the current hydrogen to metal ratio compared with the maximum saturated value (Eq. (12)), increasing the 
heat transfer coefficient means that the process finishes sooner, as the bottle is colder. Modifying the heat transfer coefficient from $10 \mathrm{~W} \mathrm{~m}^{-2} \mathrm{~K}^{-1}$ (natural convection) to $50 \mathrm{~W} \mathrm{~m}^{-2} \mathrm{~K}^{-1}$ (forced air cooling) results in the most significant improvement in the hydrogen absorption, and the improvement becomes less with further increment of the cooling level.

Whilst hydrogen fraction reaches about 0.40 under nature air cooling at $600 \mathrm{~s}$, it reaches a value of almost 1 under the other more effective conditions.

Jiao et al. [15] also studied the effects of the cooling level on the absorbed hydrogen fraction obtaining comparable results. Obviously in both cases the charging performance is more efficient if the convection coefficient increases.

These sensitivity studies show the influence of each parameter but also the changes that the modification of these parameters causes in the model. Then, keeping the simulation parameters as in Table 1, the geometry is modified to match the metal hydride canisters available in the lab (Fig. 1, b), and also the operating conditions ( $p_{\text {in, }}, T_{\text {ref }}$ and $T_{a m b}$ ) to emulate the conditions used in the experimental tests. Regarding the convection coefficient $(h)$, an estimation is made taking into account that the real application is cooled by natural convection.

Concerning metal properties, an extensive search in the literature is done to define the ranges of variation of the previously identified key properties in metal hydrides used for hydrogen storage. The absorption rate constant $\left(C_{a}\right)$ varies from 0.03 to $175.07 \mathrm{~s}^{-1}$ and the activation energy $\left(E_{a}\right)$ varies between 13000 and $49674 \mathrm{~J} \mathrm{~mol}^{-1}$. As for the thermal conductivity of the metal and the porosity, their variations range between 0.524 and $3.18 \mathrm{~W} \mathrm{~m}^{-1} \mathrm{~K}^{-1}$ and 0.3 to 0.63 respectively $[2,15,16,22,30-35]$. In the last two cases $\left(k^{m}\right.$ and $\varepsilon$ ) it has been considered that the range of variation of the parameters is small and the simulation results are not drastically affected by the selected value of these parameters, so the ones used in Table 1 are kept.

Taking all this into account, it is possible to calibrate the simulation to the real metal hydride bottles in the lab, and the new parameters are the ones shown in Table 2.

Table 2 Modified parameters for the numerical simulation with experimental conditions

\begin{tabular}{lcl}
\hline Parameter & Designation & Value \\
\hline Reference temperature & $T_{\text {ref }}$ & $297[\mathrm{~K}]$ \\
\hline Inlet pressure (relative) & $p_{\text {in }}$ & $0.65[\mathrm{MPa}]$ \\
\hline Ambient temperature & $T_{a m b}$ & $296[\mathrm{~K}]$ \\
\hline Hydrogen absorption constant on metal & $C_{a}$ & $2\left[\mathrm{~s}^{-1}\right]$ \\
\hline Hydrogen activation energy & $E_{a}$ & $27200\left[\mathrm{~J} \mathrm{~mol}^{-1}\right]$ \\
\hline Convection coefficient & $h$ & 5 or $10\left[\mathrm{~W} \mathrm{~m}^{-2} \mathrm{~K}^{-1}\right]$ \\
\hline
\end{tabular}




\subsection{Literature and experimental comparison}

\subsubsection{Literature comparison}

According to equation (1), mass conservation, the magnitude of the hydrogen gas velocity in the porous metal vessel is proportional to the rate of the hydrogen absorption reaction. Fig. 6, a) shows the gas velocity distributions, and it can be observed that at the beginning hydrogen gas velocities are high due to the initially fast hydrogen absorption reaction and then continue to decrease with time due to the slowdown in the reaction rate as hydrogen absorption proceeds [16].
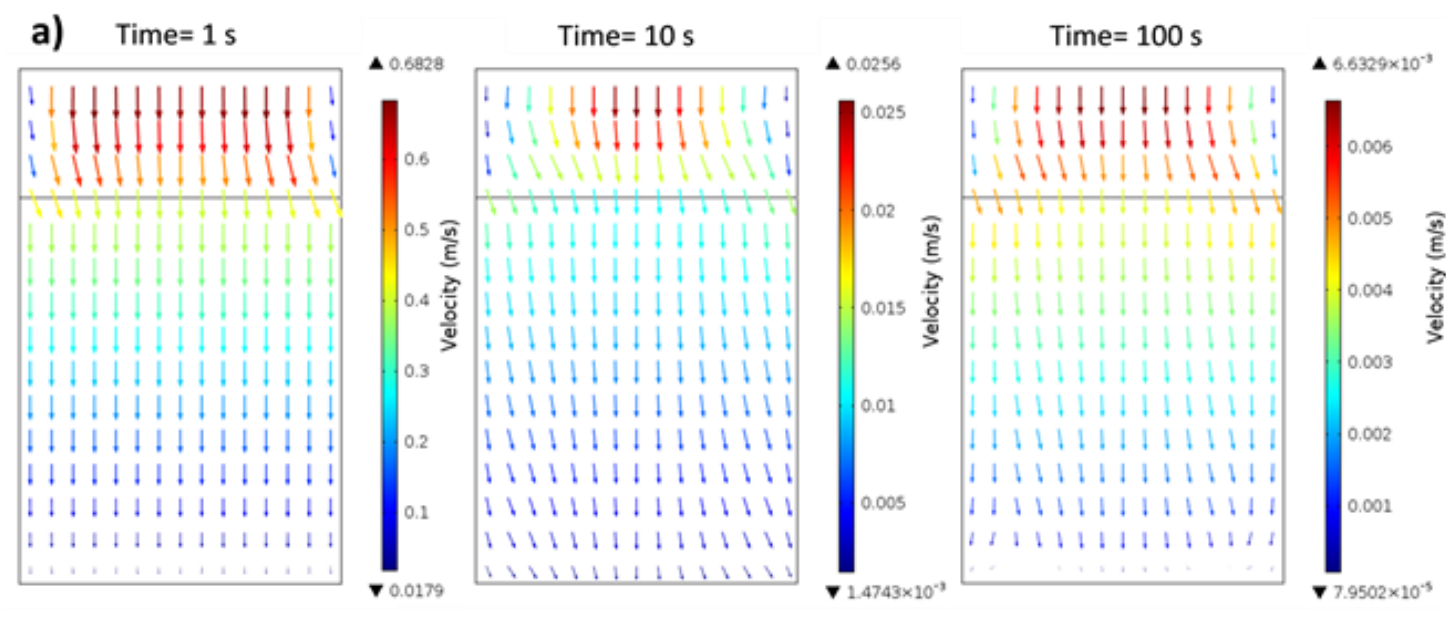

b) Time $=1 \mathrm{~s}$

Time $=10 \mathrm{~s}$
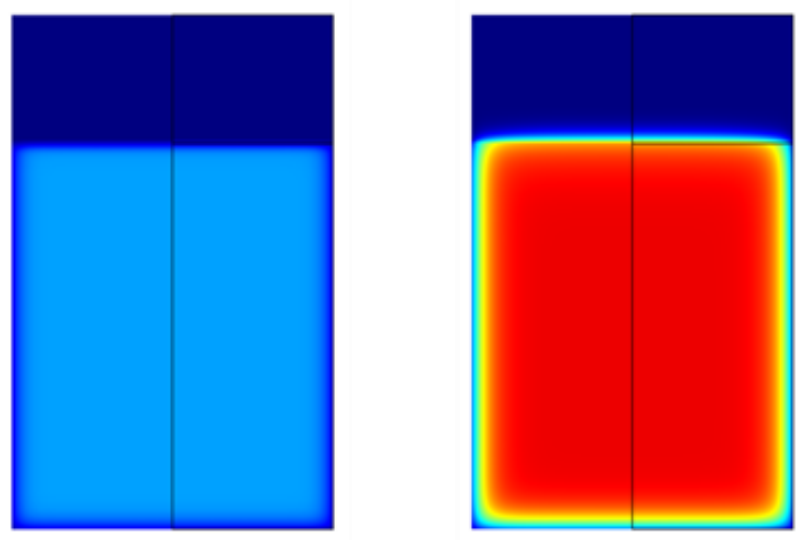

Time $=100 \mathrm{~s}$

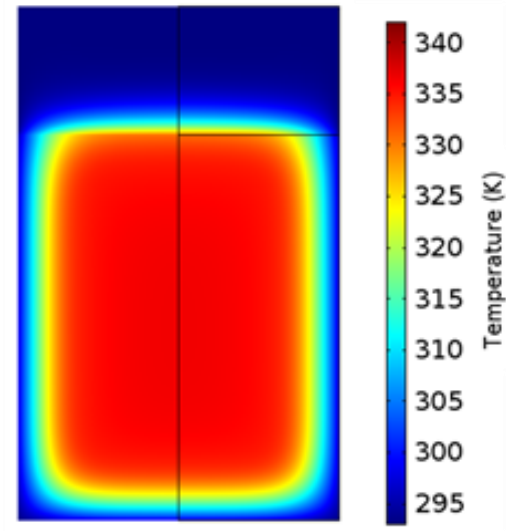

Fig. 6 a) Velocity profiles and b) Temperature contours, in a cross-section of the hydrogen storage vessel at $1 \mathrm{~s}, 10 \mathrm{~s}$, and $100 \mathrm{~s}$

On the other hand, the direction of the hydrogen gas velocity indicates the location, where hydrogen absorption is more active inside the metal hydride canister.

The hydrogen gas flows towards the vessel walls during the first $100 \mathrm{~s}$, because the temperature is lower, which means that the hydrogen absorption rate is higher near the walls. However, after $100 \mathrm{~s}$, the direction of the velocity is altered towards the core region and hydrogen absorption almost stops near the wall area. 
As can be seen by the size of the arrows, velocity decreases through the length of the bottle because hydrogen is being absorbed by the metal.

Fig. 6, a) also shows that the velocity decreases over time ( $1 \mathrm{~s}, 10 \mathrm{~s}$ and $100 \mathrm{~s})$ and finally goes to zero, as the hydrogen has already filled all the possible void volume inside the metal hydride bottle.

Nam et al. [16] show a similar graph in which it can be clearly seen that the direction of the flow is the same as in the presented simulation, indicating that the hydrogen absorption rate is higher near the walls at the beginning and then it progressively changes going towards the core region over time. Although they only provide an average velocity value for every depicted time instant, the orders of magnitude of both simulations is the same.

Fig. 6, b) provides information on temperature contours in a cross-section of the hydrogen storage vessel at $1 \mathrm{~s}, 10 \mathrm{~s}$ and $100 \mathrm{~s}$. At $t=1 \mathrm{~s}$ the bottle is at initial conditions but its temperature rapidly increases as at $t=10 \mathrm{~s}$ the bottle has reached its maximum temperature in the porous metal hydride region. At $t=100 \mathrm{~s}$, the core region is still at a high temperature but the walls are begining to cool down due to the convection with surrounding exterior air. It can also be seen that some heat is transferred to the buffer region as its temperature has increased a little in the boundary region between the buffer area and the metal hydride zone.

Fig. 6, b) can also be compared to the results obtained by Nam et al. [16]. Overall, both simulations show the same temperature contours, at the beginning the bottle is at initial conditions and then temperature increases in the core region where the exothermic reaction occurs. Values of temperaure are similar in both cases, although in this paper the transition between cold and hot zones is smoother as compared to the results obtained by Nam et al., as the quality of the mesh is improved, allowing a clearer visualization of the temperature results.
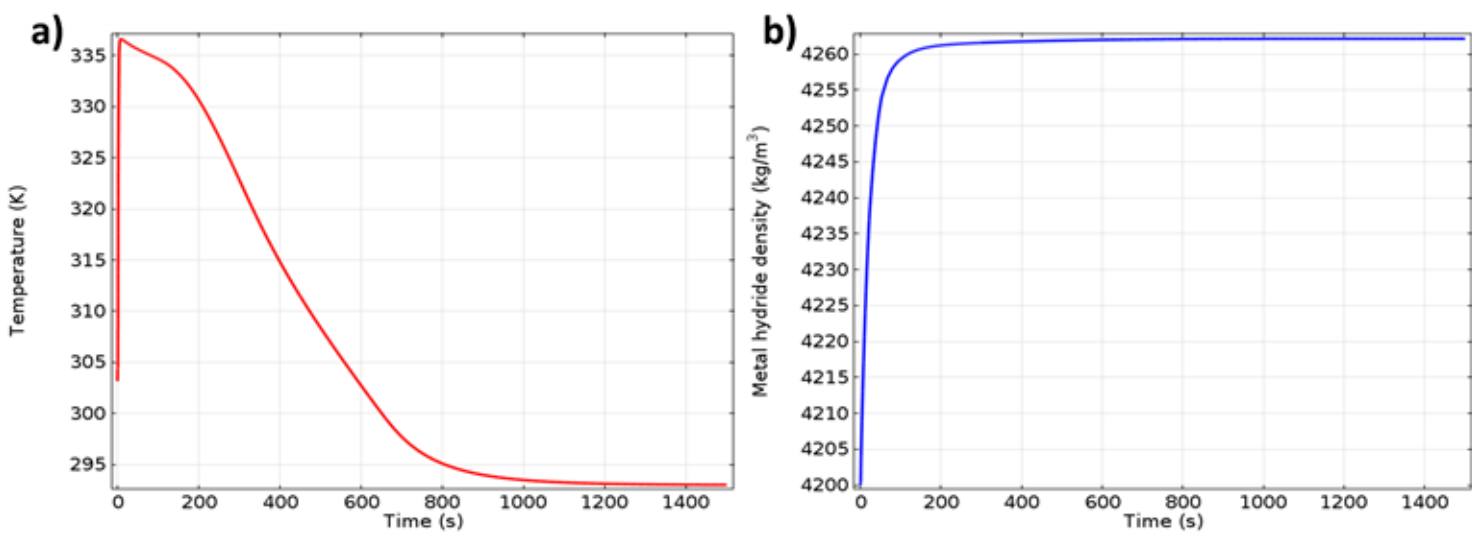

Fig. 7 a) Temperature evolution profile in a point located at $r=12 \mathrm{~mm}$ and $z=15 \mathrm{~mm}, h=1652 \mathrm{~W} \mathrm{~m}^{-2} \mathrm{~K}^{-1}$. b) Metal hydride density over time. Point located at $r=0, z=0, h=1652 \mathrm{~W} \mathrm{~m}^{-2} \mathrm{~K}^{-1}$

Focusing on the evolution of temperature in Fig. 7, a), it can be seen that the curve starts at an arbitrary initial bottle temperature $\left(T_{\text {ref }}\right)$ and in the first few seconds a sharp rise in temperatures is observed. This increase is due to the fast absorption reaction which produces heat. As it can be shown, temperature reaches its maximum value around $340 \mathrm{~K}$. 
After that, the canister starts to cool down gradually. At first a bit slower and then at a much faster rate until it reaches the temperature of the cooling fluid $\left(T_{a m b}\right)$, when it stabilizes.

Fig. 7, b), represents the metal hydride density evolution over time. It can be seen that at first metal hydride density increases very sharply, due to the initial fast absorption reaction and as a result, a high amount of hydrogen is combined with metal to form a metal hydride, hence its density increases. This sharp growth in density matches the high temperature levels (Fig. 7, a), because the absorption reaction is taking place.

Then, density slows down its growth until it saturates. The final density is achieved when the material is fully saturated.

Many authors [15-17,36] have studied the temperature evolution and the density (or absorbed hydrogen fraction) in metal hydride hydrogen storage systems all leading to similar curves that can be slightly modified depending on the parameters chosen, as demonstrated in previous sections.

\subsubsection{Comparison of simulation and experimental results}

In Fig. 8, a comparison between simulation results and experimental data for the evolution of temperature and hydrogen mass flow entering the bottle can be observed for both forced convection (fan) and natural convection (no fan) cases.

The simulation results are plotted in solid lines and have different convection coefficients, simulating natural convection $\left(h=5 \mathrm{~W} \mathrm{~m}^{-2} \mathrm{~K}^{-1}\right.$ ) and forced convection of air applied on the surface of the bottle $\left(h=10 \mathrm{~W} \mathrm{~m}^{-2} \mathrm{~K}^{-1}\right)$. The experimental results are represented in asterisks being the black ones the result of forced convection and the red natural convection.
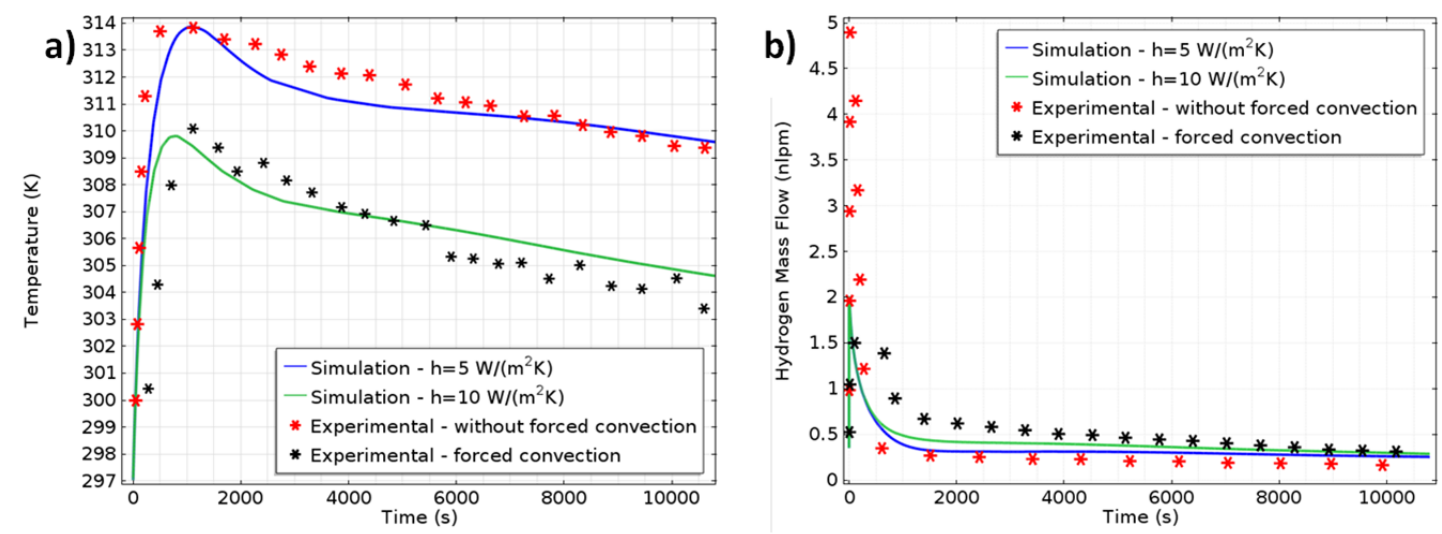

Fig. 8 Simulation and experimental results with natural and forced convection of: a) Temperature in a point located $r=20 \mathrm{~mm}, \mathrm{z}=80 \mathrm{~mm}$. b) Hydrogen mass flow in a point located $r=0, z=273 \mathrm{~mm}$

As can be seen in Fig. 8, a), the experimental and simulation results based on the temperature evolution, match closely. Focusing on the forced convection case (green line and black asterisks) it can be determined that the numerical method model is able to emulate the evolution of bottle surface temperature, as the simulation curve is very similar to the experimental results, including the shape of the curve, the temperature peak and most importantly, the tendency. 
In Fig. 8, b) a comparison between experimental and simulation results based on the hydrogen mass flow is shown. It can be observed that the simulation results match the experimental data for the most part, but there are some differences basically on the initial peak. It has to be taken into account that the MFC is placed outside the bottle, so it is measuring the amount of hydrogen entering the canister (inlet mass flow) whereas simulation results indicate the hydrogen mass flow being absorbed (absorbed mass flow).

Comparing natural and forced convection results, as expected, if the bottle is not cooled externally, its temperature will be higher due to the exothermal reaction. If the bottle is hot, hydrogen gas molecules will be more excited and as a consequence they will create higher local pressure inside the bottle. In this case, the pressure gradient from the inlet to the interior of the bottle will be smaller, causing the hydrogen mass flow at the inlet to be reduced.

It is also important to mention that the first experiments (natural convection) are performed using a MFC with a range that goes from 0 to $5 \mathrm{nlpm}$ but in the other case (forced convection), the experiments were performed using a MFC with a range that goes from 0 to $1.5 \mathrm{nlpm}$, so the hydrogen mass flow in the first instants is limited to $1.5 \mathrm{nlpm}$, but when it decreases all the data is acquired and, as observed, matches the simulation results.

\section{Conclusions}

A mathematical model, which rigorously accounts for the principles of mass, momentum and energy conservation, and absorption kinetics, has been developed to simulate the hydrogen absorption process in a metal hydride tank. The effects of metal properties as well as cooling level have been numerically simulated as well.

First, the validity of this model was tested by comparison with other author's published work, achieving a good agreement between all the data.

This model was then adapted to match the real laboratory conditions, by means of adjusting the bottle shape and size, reducing the convective heat transfer coefficient compared to published works $[15,16]$ between the container and the surrounding air, and also modifying the metal properties, which was possible to do because of exhaustive experimental testing and analysis of the sensitivity of the model to metal properties.

It has been determined that the metal properties that most influence the charging performance are the absorption rate constant $(\mathrm{Ca})$, the activation energy ( $\mathrm{Ea}$ ) and the thermal conductivity $(\mathrm{km})$, as they have a considerable influence on the bottle temperature. The porosity $(\varepsilon)$ has also a strong effect on the charge performance. If porosity increases, the amount of hydrogen that can be stored is lower, and so the absorption process finishes sooner. Given the impact of bottle temperature on the charging performance, the external cooling level has been studied as well, determining that if the convection coefficient is higher (cooler bottle), charging process is faster.

In addition, an experimental setup using commercially available metal hydride bottles was designed, assembled and a data acquisition system using a NI myRIO device was programmed on LabVIEW, in order to obtain the required experimental data to be compared with the numerical model. 
The simulation results show the same tendencies in comparison with experimental data, and so it is demonstrated that the model successfully captures the essential experimental trends that can be observed. Moreover, the detailed numerical and experimental results highlight an initial increase in the vessel's temperature as well as in the metal hydride density and also faster hydrogen gas velocities inside the bottle due to the quick hydrogen absorption rate at the beginning. However, towards the end of the absorption process, the effect of the cooling level becomes a great influence on controlling the amount of hydrogen entering the bottle, and thus the charging time [28].

The results and experimental data obtained in this paper can be used to assess the state of charge of the bottle as a future work, but also as a procedure for monitoring the current status of the bottle and the supervision of the bottle's performance, evaluating aspects such as degradation of the metal hydride material due to fatigue or wear, reduction of the hydrogen absorption capacity of the metal due to contamination (for example presence of air and water vapor).

\section{Acknowledgements}

All the experimental tests were performed at the PEM Fuel Cells Laboratory of the Institut de Robòtica i Informàtica Industrial (CSIC-UPC, Barcelona, Spain) and was only possible due to its advanced equipment and proficient technical staff. This work has been partially funded by the Spanish national project MICAPEM (ref. DPI2015-69286-C3-2-R, MINECO/FEDER), and the Spanish Council for Scientific Investigations JAE-INTRO2014.

\section{References}

[1] Sharaf OZ, Orhan MF. An overview of fuel cell technology: Fundamentals and applications. Renew Sustain Energy Rev 2014;32:810-53. doi:10.1016/j.rser.2014.01.012.

[2] Muthukumar P, Singhal A, Bansal GK. Thermal modeling and performance analysis of industrial-scale metal hydride based hydrogen storage container. Int J Hydrogen Energy 2012;37:14351-64. doi:10.1016/j.ijhydene.2012.07.010.

[3] Dhaou H, Mellouli S, Askri F, Jemni A, Ben Nasrallah S. Experimental and numerical study of discharge process of metal-hydrogen tank. Int J Hydrogen Energy 2007;32:1922-7. doi:10.1016/j.ijhydene.2006.08.045.

[4] Rusman NAA, Dahari M. A review on the current progress of metal hydrides material for solid-state hydrogen storage applications. Int J Hydrogen Energy 2016;41:12108-26. doi:10.1016/j.ijhydene.2016.05.244.

[5] Dornheim M. Thermodynamics of Metal Hydrides: Tailoring Reaction Enthalpies of Hydrogen Storage Materials. Thermodyn - Interact Stud - Solids, Liq Gases, 2011:891918. doi:10.5772/21662.

[6] Patil SD, Ram Gopal M. Analysis of a metal hydride reactor for hydrogen storage. Int J Hydrogen Energy 2013;38:942-51. doi:10.1016/j.ijhydene.2012.10.031.

[7] Akanji O, Kolesnikov A. Modeling and Simulation of Hydrogen Storage Device for Fuel Cell Using COMSOL Multiphysics. COMSOL Conf. 2011 Stuttgart, 2011, p. 1-26.

[8] Sakintuna B, Lamari-Darkrim F, Hirscher M. Metal hydride materials for solid hydrogen storage: A review. Int J Hydrogen Energy 2007;32:1121-40. doi:10.1016/j.ijhydene.2006.11.022. 
[9] Barbir F. PEM Fuel Cells. Theory and Practice. 2005.

[10] Jemni A, Ben Nasrallah S. Study of two-dimensional heat and mass transfer during absorption in a metal-hydrogen reactor. Int J Hydrogen Energy 1995;20:43-52. doi:10.1016/0360-3199(93)E0007-8.

[11] Jemni a, Nasrallah S Ben. Study of two-dimensional heat and mass transfer during desorption in ametal-hydrogen reactor. Int J Hydrogen Energy 1995;20:881-91. doi:10.1016/0360-3199(94)00115-G

[12] Jemni A, Ben Nasrallah S, Lamloumi J. Experimental and theoretical study of a metalhydrogen reactor. Int J Hydrogen Energy1 1999;24:631-44. doi:10.1016/S03603199(98)00117-7.

[13] Muthukumar P, Madhavakrishna U, Dewan A. Parametric studies on a metal hydride based hydrogen storage device. Int J Hydrogen Energy 2007;32:4988-97. doi:10.1016/j.ijhydene.2007.08.010.

[14] Satya Sekhar B, Muthukumar P, Saikia R. Tests on a metal hydride based thermal energy storage system. Int J Hydrogen Energy 2012;37:3818-24. doi:10.1016/j.ijhydene.2011.05.114.

[15] Jiao K, Li X, Yin Y, Zhou Y, Yu S, Du Q. Effects of various operating conditions on the hydrogen absorption processes in a metal hydride tank. Appl Energy 2012;94:257-69. doi:10.1016/j.apenergy.2012.01.033.

[16] Nam J, Ko J, Ju H. Three-dimensional modeling and simulation of hydrogen absorption in metal hydride hydrogen storage vessels. Appl Energy 2012;89:164-75. doi:10.1016/j.apenergy.2011.06.015.

[17] Chung CA, Ho CJ. Thermal-fluid behavior of the hydriding and dehydriding processes in a metal hydride hydrogen storage canister. Int J Hydrogen Energy 2009;34:4351-64. doi:10.1016/j.ijhydene.2009.03.028.

[18] Dhaou H, Askri F, Ben Salah M, Jemni A, Ben Nasrallah S, Lamloumi J. Measurement and modelling of kinetics of hydrogen sorption by LaNi5 and two related pseudobinary compounds. Int J Hydrogen Energy 2007;32:576-87. doi:10.1016/j.ijhydene.2006.07.001.

[19] Souahlia A, Dhaou H, Mellouli S, Askri F, Jemni A, Ben Nasrallah S. Experimental study of metal hydride-based hydrogen storage tank at constant supply pressure. Int J Hydrogen Energy 2014;39:7365-72. doi:10.1016/j.ijhydene.2014.02.121.

[20] Baldissin D, Lombardo D. Thermofluidynamic Modelling of Hydrogen Absorption and Desorption in a LaNi4.8AI0.2 Hydride Bed. COMSOL Conf. 2009 Milan, 2009.

[21] Jemni A, Nasrallah S Ben, Lamloumi J, Guégan a. P. Study of Heat and Mass Transfer in a Metal - Hydrogen Reactor. Zeitschrift Für Phys Chemie 1994;183:197-203. doi:10.1524/zpch.1994.183.Part_1_2.197.

[22] Bao Z, Wu Z, Nyamsi SN, Yang F, Zhang Z. Three-dimensional modeling and sensitivity analysis of multi-tubular metal hydride reactors. Appl Therm Eng 2013;52:97-108. doi:10.1016/j.applthermaleng.2012.11.023.

[23] Talagañis BA, Meyer GO, Aguirre PA. Modeling and simulation of absorption-desorption cyclic processes for hydrogen storage-compression using metal hydrides. Int J Hydrogen Energy 2011;36:13621-31. doi:10.1016/j.ijhydene.2011.07.139.

[24] COMSOL. COMSOL Multiphysics Version 4.3a Documentation, Subsurface Fluid Flow Module 2012:1-16.

[25] Truskey GA, Yuan F, Katz DF. Transport Phenomena in Biological Systems. Pearson 
Prentice Hall; 2004.

[26] Nield D, Bejan A. Convection in Porous Media. Springer-Verlag New York; 2006. doi:10.1007/0-387-33431-9.

[27] Florida Solar Energy Center (FSEC). Metal Hydride Storage 2005.

[28] Busqué R, Torres R, Husar AP. Metal Hydride State Of Charge Estimator. Development and experimental validation of the hydrogen storage system model. UPC-CSIC, 2015.

[29] Strahl S. Characteritzation, development and experimental validation of dynamic PEM fuel cell system model. UPC-CSIC, 2007.

[30] Ma J, Wang Y, Shi S, Yang F, Bao Z, Zhang Z. Optimization of heat transfer device and analysis of heat $\&$ mass transfer on the finned multi-tubular metal hydride tank. Int J Hydrogen Energy 2014;39:13583-95. doi:10.1016/j.jjhydene.2014.03.016.

[31] Meng X, Bao Z, Wu Z, Yang F, Zhang Z. A Comprehensive Performance Evaluation Index for Metal Hydrides Reactor. Energy Procedia 2012;29:421-30. doi:10.1016/j.egypro.2012.09.049.

[32] Ben Mâad H, Askri F, Ben Nasrallah S. Numerical investigation of heat and mass transfer during the desorption process of an $\mathrm{Mg} 2 \mathrm{Ni}-\mathrm{H} 2$ reactor. Int J Hydrogen Energy 2013;38:4597-610. doi:10.1016/j.ijhydene.2013.01.144.

[33] Yoo H, Ko J, Yun S, Chang M, Kang H, Kim W, et al. A numerical investigation of hydrogen desorption phenomena in ZrCo based hydrogen storage beds. Int J Hydrogen Energy 2013;38:6226-33. doi:10.1016/j.ijhydene.2012.12.098.

[34] Visaria M, Mudawar I. Experimental investigation and theoretical modeling of dehydriding process in high-pressure metal hydride hydrogen storage systems. Int J Hydrogen Energy 2012;37:5735-49. doi:10.1016/j.ijhydene.2011.12.140.

[35] Lee M, Kim KJ, Hopkins RR, Gawlik K. Thermal conductivity measurements of coppercoated metal metal hydride hydrogen compression systems. Int J Hydrogen Energy 2009;34:3185-90. doi:10.1016/j.ijhydene.2009.01.078.

[36] Ben Mâad H, Miled A, Askri F, Ben Nasrallah S. Numerical simulation of absorptiondesorption cyclic processes for metal-hydrogen reactor with heat recovery using phasechange material. Appl Therm Eng 2016;96:267-76. doi:10.1016/j.applthermaleng.2015.11.093. 